\title{
A Lateralized Brain Network for Visuospatial Attention
}

Michel Thiebaut de Schotten ${ }^{* 1,2,3 \dagger}$, Flavio Dell'Acqua ${ }^{1,3,4 \dagger}$, Stephanie Forkel ${ }^{1}$, Andrew Simmons ${ }^{3,4,5}$, Francesco Vergani ${ }^{6}$, Declan G.M. Murphy ${ }^{1}$ and Marco Catani 1,3

${ }^{1}$ Natbrainlab, Department of Forensic and Neurodevelopmental Sciences, Institute of Psychiatry, King's College London, London, UK

${ }^{2}$ INSERM-UPMC UMR S 975, G.H. Pitié-Salpêtrière, Paris, France

${ }^{3}$ Department of Neuroimaging, Institute of Psychiatry, King's College London, London, UK

${ }^{4}$ NIHR Biomedical Research Centre for Mental Health at South London and Maudsley NHS Foundation Trust and King's College London Institute of Psychiatry, $U K$

${ }^{5}$ MRC Centre for Neurodegeneration Research, King's College London, UK

${ }^{6}$ Department of Neurosurgery, Royal Victoria Infirmary, Newcastle upon Tyne, UK

${ }^{\dagger}$ Contributed Equally to this work

*Corresponding Author: Michel Thiebaut de Schotten, Natbrainlab, Department of Forensic and Neurodevelopmental Sciences, King's College, Institute of Psychiatry, 16 De Crespigny Park, SE5 8AF London UK. Email: michel.thiebaut@gmail.com 


\begin{abstract}
Right hemisphere dominance for visuospatial attention is characteristic for most humans, but its anatomical basis remains unknown. We report the first evidence in humans for a larger parieto-frontal network in the right than left hemisphere; and a significant correlation between the degree of anatomical lateralization and asymmetry of performance on visuospatial tasks. Our results suggest that hemispheric specialization is associated with an unbalanced speed of visuospatial processing.
\end{abstract}


Clinical studies in patients with brain lesions, and decades of neuropsychological testing in split-brain patients, have led to the assumption that visuospatial attention is a function of the right hemisphere ${ }^{1,2}$. Nevertheless, right visuospatial neglect is not an infrequent finding in patients with left hemisphere damage ${ }^{3}$ suggesting that visuospatial attention is probably a bilateral function; with right hemisphere dominance in most, but not all, humans ${ }^{4}$. However, the anatomical basis of the hemispheric dominance for visuospatial attention is largely unknown.

In the monkey brain the activity of neurons dedicated to visuospatial attention has been recorded simultaneously using multiple electrodes implanted in the parietal and frontal cortex ${ }^{5}$. Axonal tracing studies have shown that these neurons are directly linked through a system of connections running longitudinally in the dorsolateral regions of the brain ${ }^{6}$. Recent structural $^{7}$ and functional ${ }^{8}$ imaging studies provide indirect evidence of a similar system in the human brain. However, the hemispheric lateralization of the parieto-frontal connections in the human brain and its correlation with visuospatial performances has never been demonstrated.

In this study, we performed virtual in vivo dissections of the parieto-frontal connections in 20 right-handed subjects (11 males, 9 females) using diffusion imaging tractography based on spherical deconvolution ${ }^{9}$.

A comparison between our human tractography dissections and the corresponding reconstructions from a monkey atlas $^{6}$ that we modified are illustrated in Fig. 1. Overall, parieto-frontal connections of the human and the monkey brain are similarly organized in three longitudinal parieto-frontal tracts separated into a dorsal superior 
longitudinal fasciculus (SLF) I, middle SLF II and ventral SLF III (see supplementary material) $)^{10}$.

By measuring the volumes of the tracts in both hemispheres we were able to show a dorsal to ventral gradient of lateralization of the SLF (Fig. 2a). The SLF I is symmetrically distributed between left and right hemispheres $\left(t_{(19)}<1\right)$; the SLF II shows a trend of right lateralization $\left(\mathrm{t}_{(19)}=1.141 ; p=0.268\right)$ and the SLF III is right lateralized $\left(\mathrm{t}_{(19)}=6.083 ; p<0.001\right)$.

To test whether this lateralization was related to the attentional bias for one visual hemi-field, we asked the participants to perform a line bisection test ${ }^{11}$. In the general population a small left deviation in the line bisection test is observed and referred to as the 'pseudoneglect effect' ${ }^{11}$. Consistent with previous studies ${ }^{12}$, our participants deviated towards the left at a group level $\left(-1.53 \pm 2.02 \mathrm{~mm} ; \mathrm{t}_{(19)}=3.148 ; p=0.005\right)$. In the majority of participants the correlation analysis indicates that larger SLF II volumes on the right hemisphere corresponded to a greater deviation to the left in the line bisection $(\mathrm{r}=-0.734 ; p<0.001)$ (Fig. 2b). Importantly the three subjects deviating to the right showed an opposite pattern of lateralization (i.e. larger volume of the left SLF II). Correlations with the SLF I $(r=0.258 ; p=0.286)$ and the SLF III $(\mathrm{r}=-0.295 ; p=0.220)$ were not statistically significant.

It is unknown how differences between the two hemispheres in SLF II volume can lead to asymmetrical processing of visual scenes. Larger tract in the right hemisphere could depend on a number of factors, including greater fiber myelination, higher number of axons and larger axonal diameter that are correlated with the conduction speed $^{13,14}$. In light of these previous works, we suggest that the left deviation in the 
line bisection we observed in the majority of subjects may result from unbalanced speed of visuospatial processing between the two hemispheres along the SLF II. To test this hypothesis, subjects were asked to complete a modified Posner paradigm ${ }^{15}$ in which they detected as quickly as possible targets that appeared either in their left or right hemi-field. In the majority of subjects the correlation analysis indicates that larger SLF II volumes in the right hemisphere corresponded to faster detection times in the left hemi-field $(\mathrm{r}=-0.471 ; p=0.042)$ (Fig. 2c). Correlations with the SLF I ( $\mathrm{r}$ $=0.271 ; p=0.262)$ or SLF III $(\mathrm{r}=-0.271 ; p=0.262)$ were not statistically significant. Moreover, larger deviation towards the left in the line bisection corresponded to faster detection times in the left hemi-field $(\mathrm{r}=0.495 ; p=$ 0.031)(Fig. 2d).

The correlations between the lateralization of the SLF II, the deviation observed in the line bisection and the detection times represent the first demonstration that anatomical asymmetry of the parieto-frontal connections predicts behavioral performance on visuospatial attention tasks. These findings may help to interpret the neuroanatomical basis of current models of visuospatial attention based on functional imaging and studies of neglect patients. The cortical projections of the SLF I overlap with the dorsal network activated during the voluntary orienting of spatial attention towards visual targets; and the SLF III overlaps with the ventral network that is activated during the automatic capture of spatial attention by visual targets ${ }^{8}$ and damaged in people with visuospatial neglect (supplementary figure 8). In contrast, the SLF II overlaps with the parietal component of the ventral network and the prefrontal component of the dorsal network. Thus, our findings suggest that the SLF II 
represents a direct communication between the dorsal and the ventral networks during the orienting of attention. This communication may play the role of a modulator for the dorsal network, redirecting goal directed attention mediated by the SLF I to events identified as salient by the SLF III $^{8}$.

In conclusion our study is the first to report the existence of a bilateral parieto-frontal network, previously described only in monkey, whose hemispheric lateralization predicts the degree of specialization of the right hemisphere for visuospatial attention. Our results also suggest that this hemispheric specialization is associated with an unbalanced speed of visuospatial processing along the SLF II. This lateralization may be predictive of visuospatial recovery in patients with lesions of parieto-frontal networks.

\section{REFERENCES}

1. Sperry, R.W. (Rockefeller Univ. Press, New York, 1974).

2. Mesulam, M.M. Ann. Neurol. 10, 309-325 (1981).

3. Beis, J.M., et al. Neurology 63, 1600-1605 (2004).

4. Heilman, K.M. \& Van Den Abell, T. Neurology 30, 327-330 (1980).

5. $\quad$ Buschman, T.J. \& Miller, E.K. Science 315, 1860-1862 (2007).

6. Schmahmann, J.D. \& Pandya, D.N.(Oxford Univ. Press, New York, 2006).

7. Makris, N., et al. Cereb. Cortex 15, 854-869 (2005).

8. Corbetta, M. \& Shulman, G.L. Nat. Rev. Neurosci. 3, 201-215 (2002).

9. Dell'acqua, F., et al. Neuroimage 49, 1446-1458 (2010).

10. Petrides, M. \& Pandya, D.N. J. Comp. Neurol. 228, 105-116 (1984).

11. Bowers, D. \& Heilman, K.M. Neuropsychologia 18, 491-498 (1980).

12. Jewell, G. \& McCourt, M.E. Neuropsychologia 38, 93-110 (2000).

13. Hursh, J.B. American Journal of Physiology 127, 131-139 (1939).

14. Waxman, S.G. \& Bennett, M.V. Nat. New. Biol. 238, 217-219 (1972).

15. Posner, M.I. Q. J. Exp. Psychol. 32, 3-25 (1980). 


\section{CAPTIONS}

Figure 1: Comparison between post-mortem axonal tracing in monkey (a) ${ }^{6,10}$ and in vivo SD tractography in humans (b).

Figure 2: Hemispheric lateralization of the three SLF branches with 95\% confidence intervals (a). Correlations between the lateralization of the SLF II and both the deviation on the line bisection task (b) and the lateralization of the detection time (c). Correlation between the deviation on the line bisection task and the detection time (D). ${ }^{*} \mathrm{p}<0.05$ and $* * * p<0.001$. 
Acknowledgments. We would like to thank the NATBRAINLAB

(http://www.natbrainlab.com), D. ffytche, P. Bartolomeo and F. Doricchi for discussion. This work was supported by the Marie Curie Intra-European Fellowships for Career Development (FP7), the Bettencourt-Schueller Foundation, the Guy's and St Thomas Charity, the Wellcome Trust, the NIHR Biomedical Research Centre for Mental Health at the South London and Maudsley NHS Foundation Trust and Institute of Psychiatry, Kings College and the Agence Nationale de la Recherche (ANR) [project CAFORPFC, number ANR-09-RPDOC-004-01 and project HMTC, number ANR-09-EMER-006].

Author Contributions. MTS conceived and coordinated the study, reviewed and collected neuropsychological data, performed the tractography dissections, helped with the post-mortem dissections and wrote the manuscript. FD collected and preprocessed the neuroimaging data before the dissections and helped drafting the manuscript. SF helped collecting neuropsychological data and drafting the manuscript. AS and DGM provided funding for the neuroimaging data and helped to draft the manuscript. FV helped drafting the manuscript and performed the postmortem dissections. MC helped to conceive and coordinate the study. MC also wrote the manuscript and performed the post-mortem dissections. 


\section{a: Monkey}
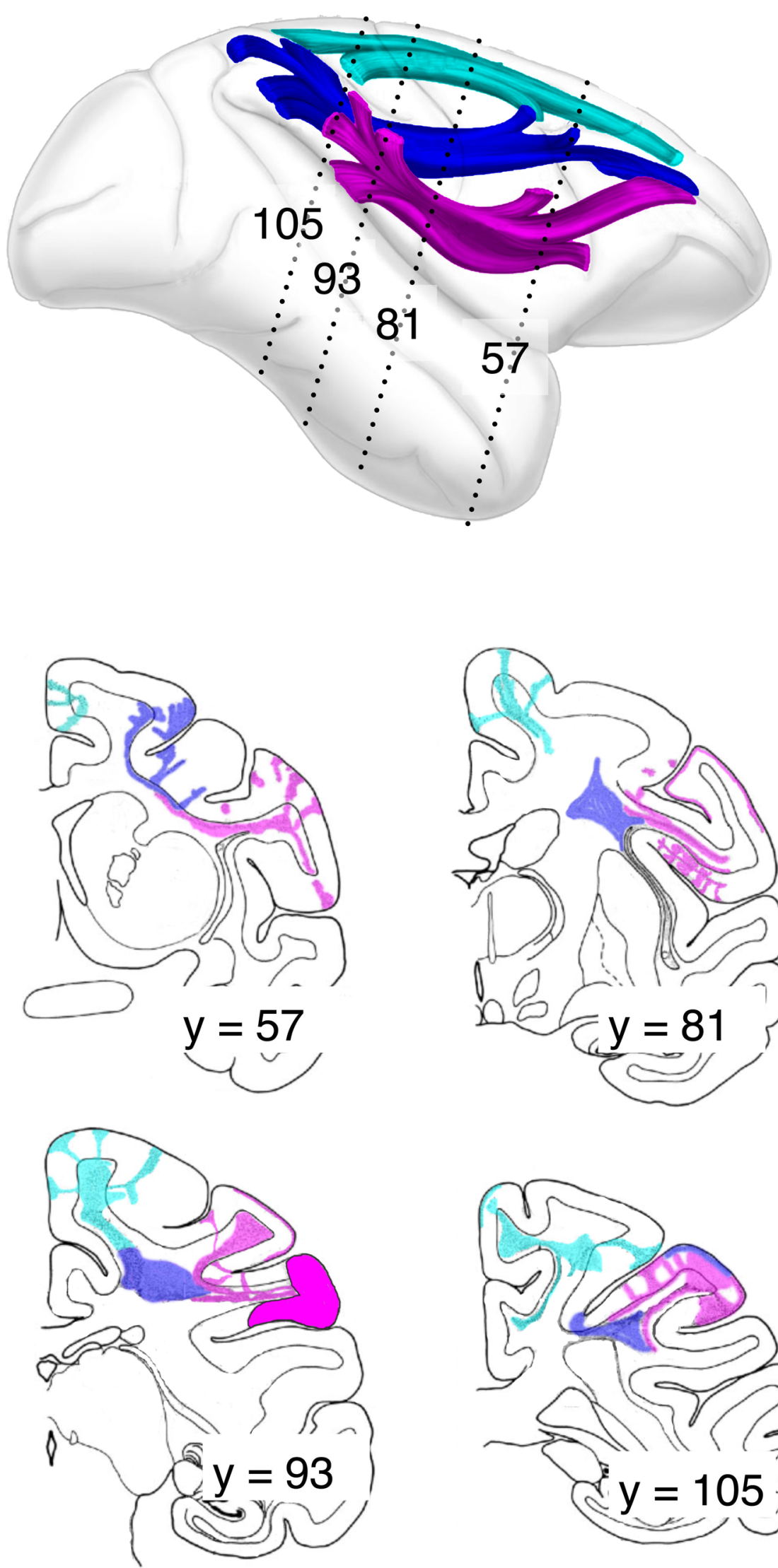

b: Human
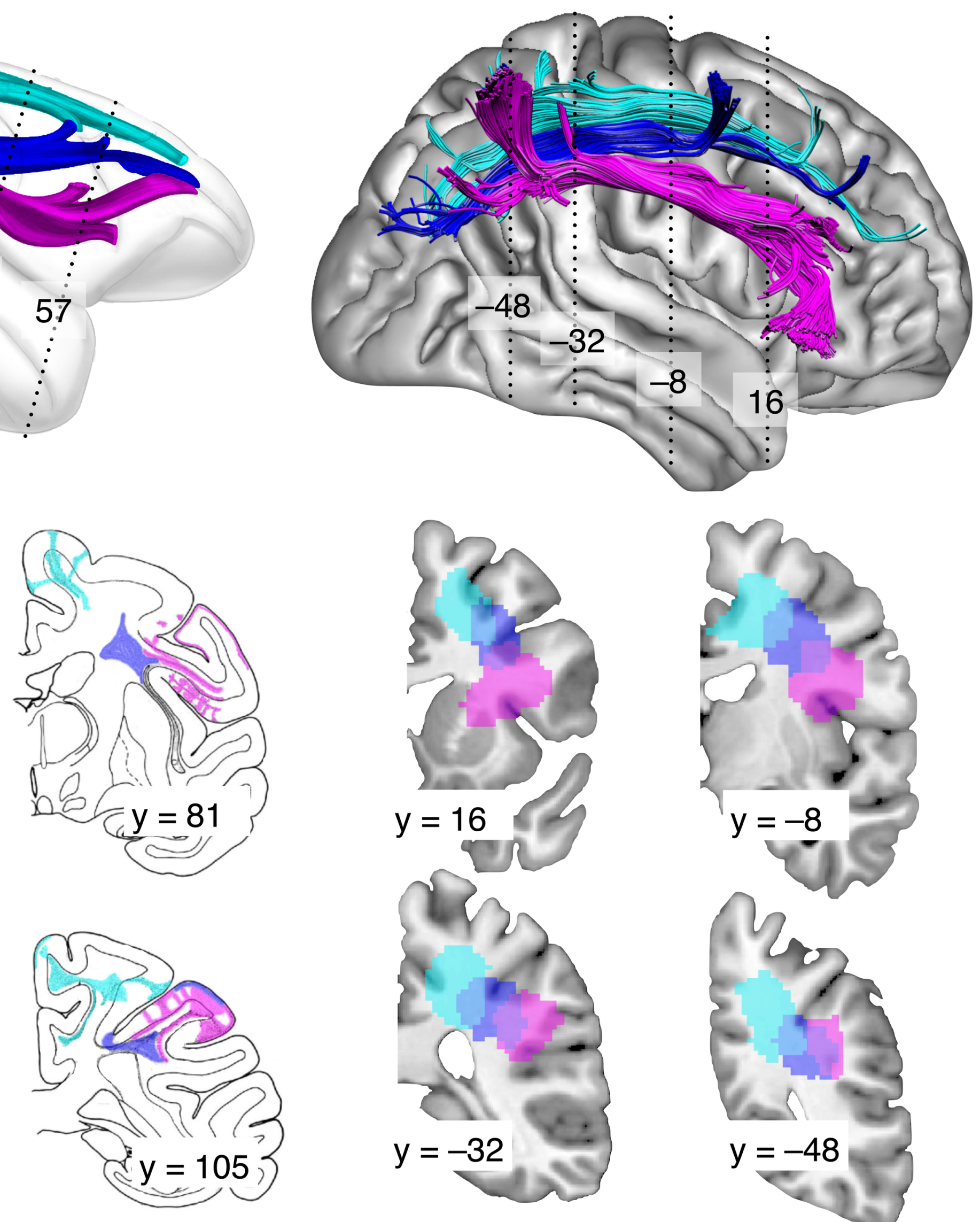
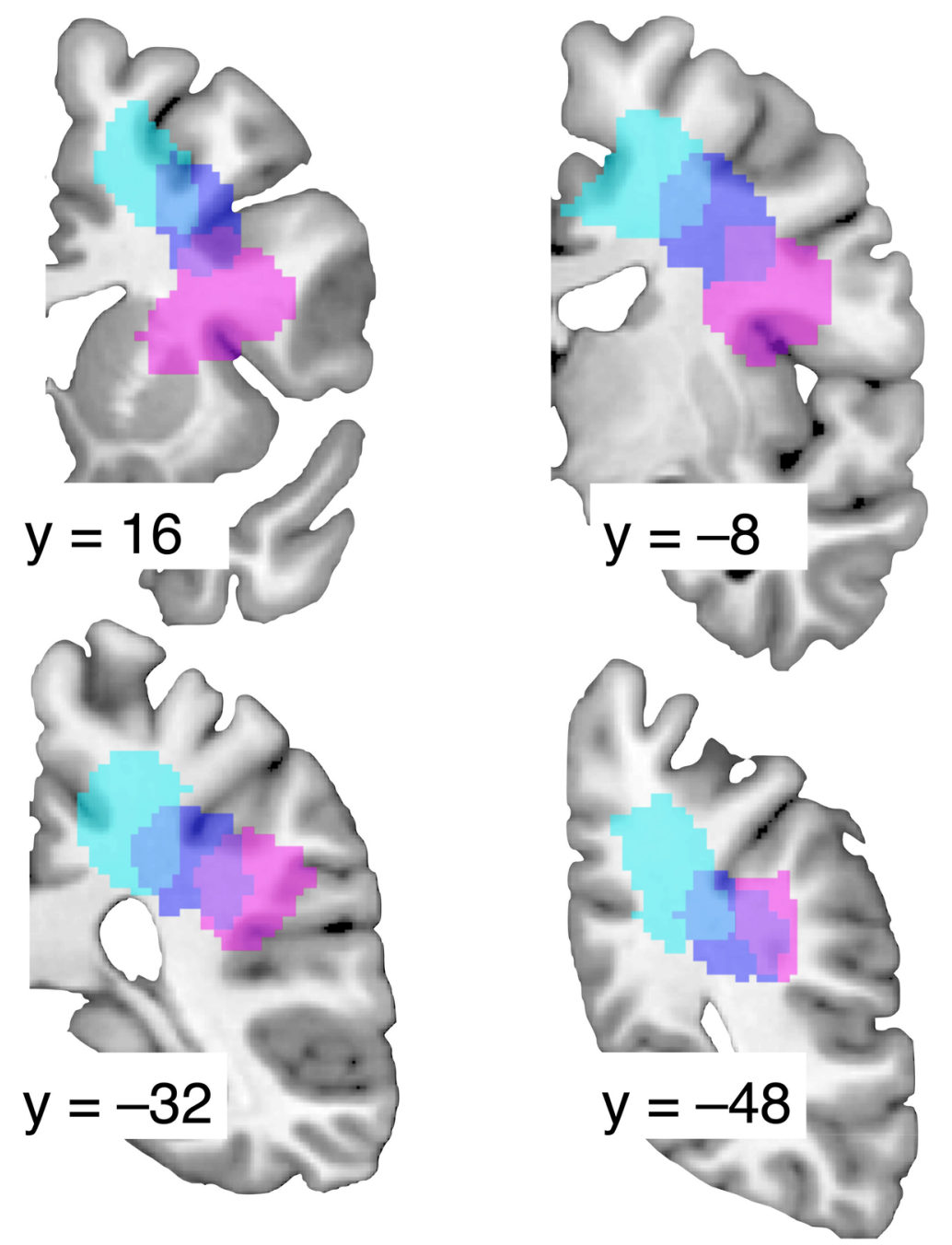

SLF I

SLF II

SLF III 
a

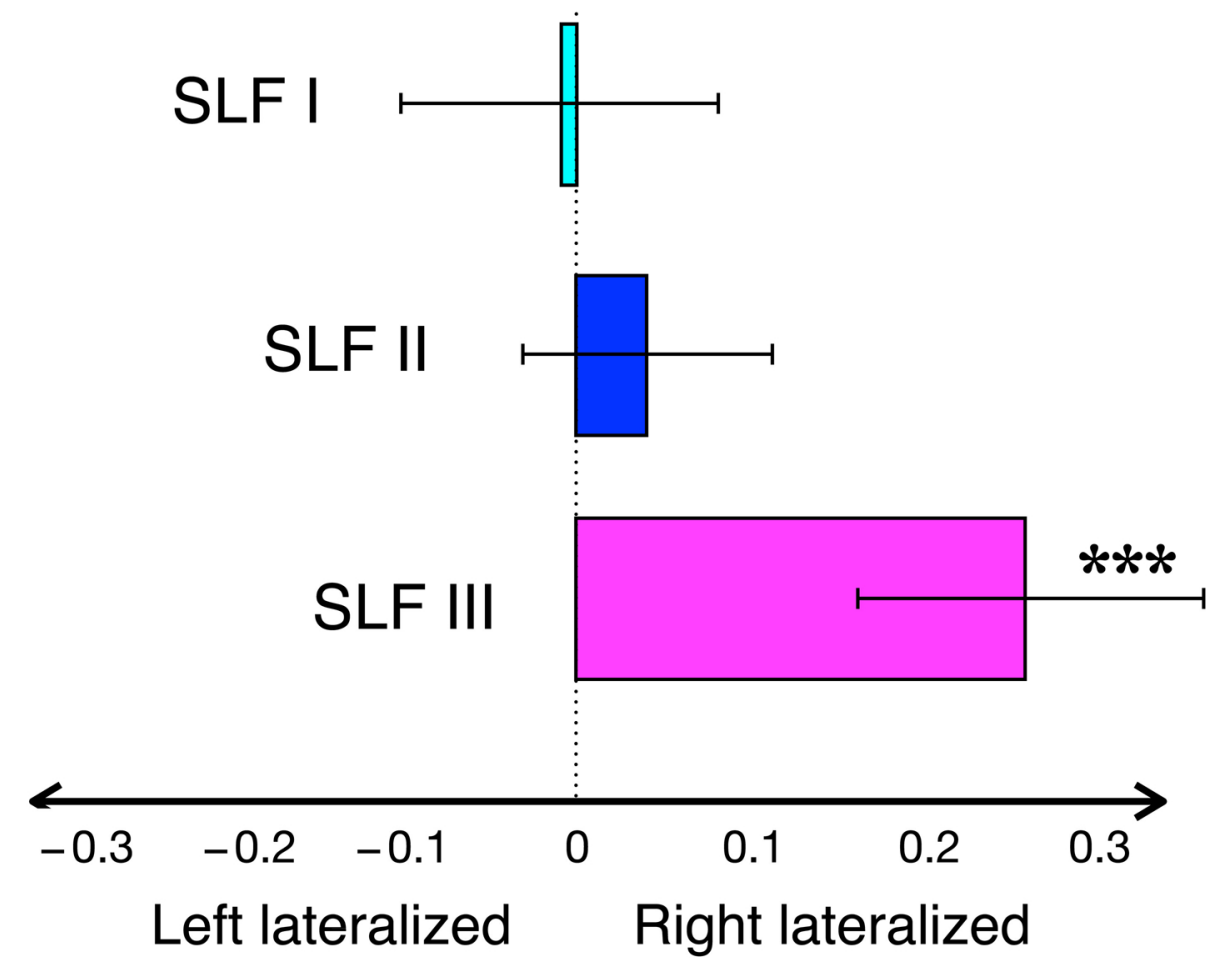

Lateralization index (volume)

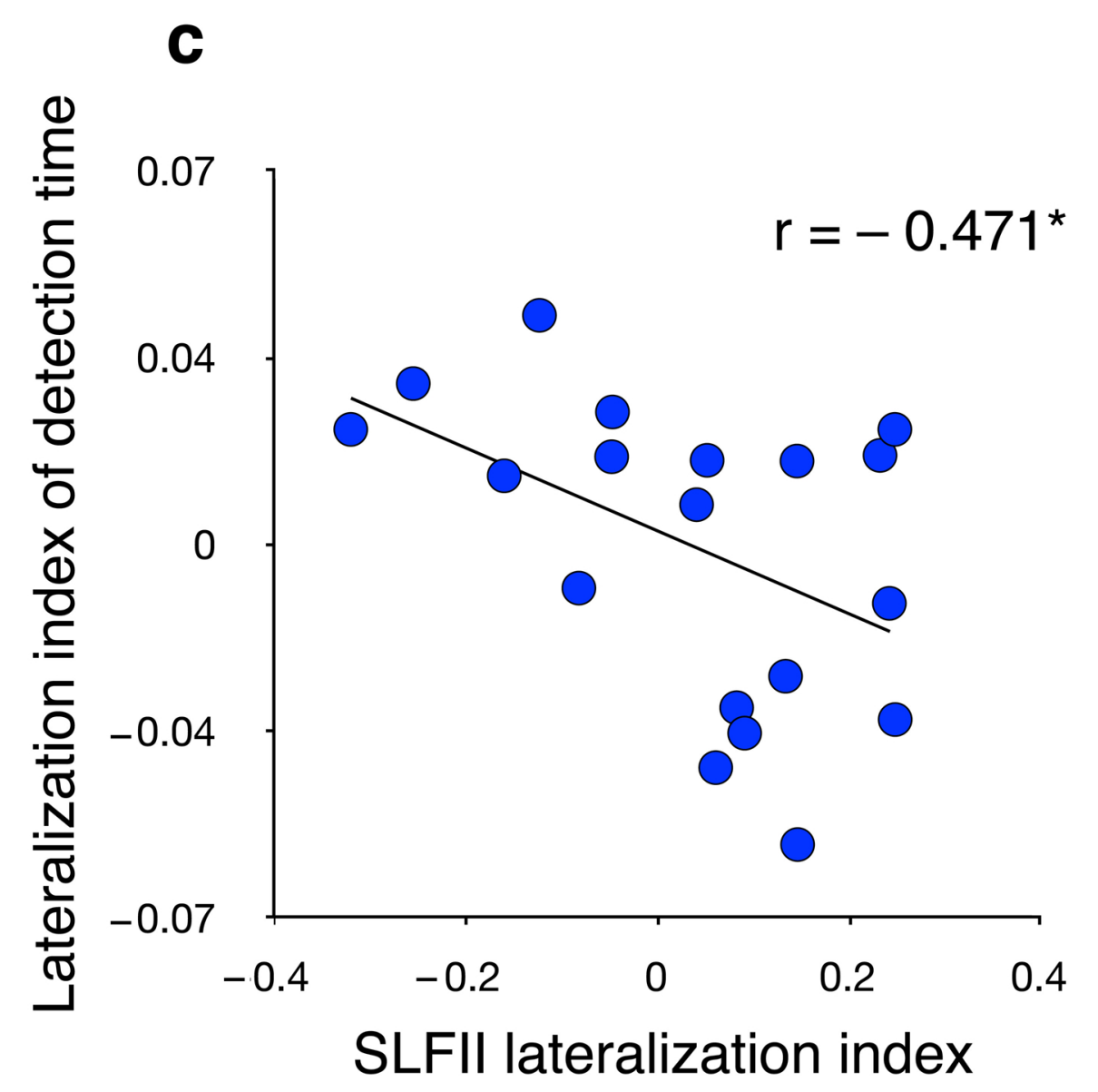

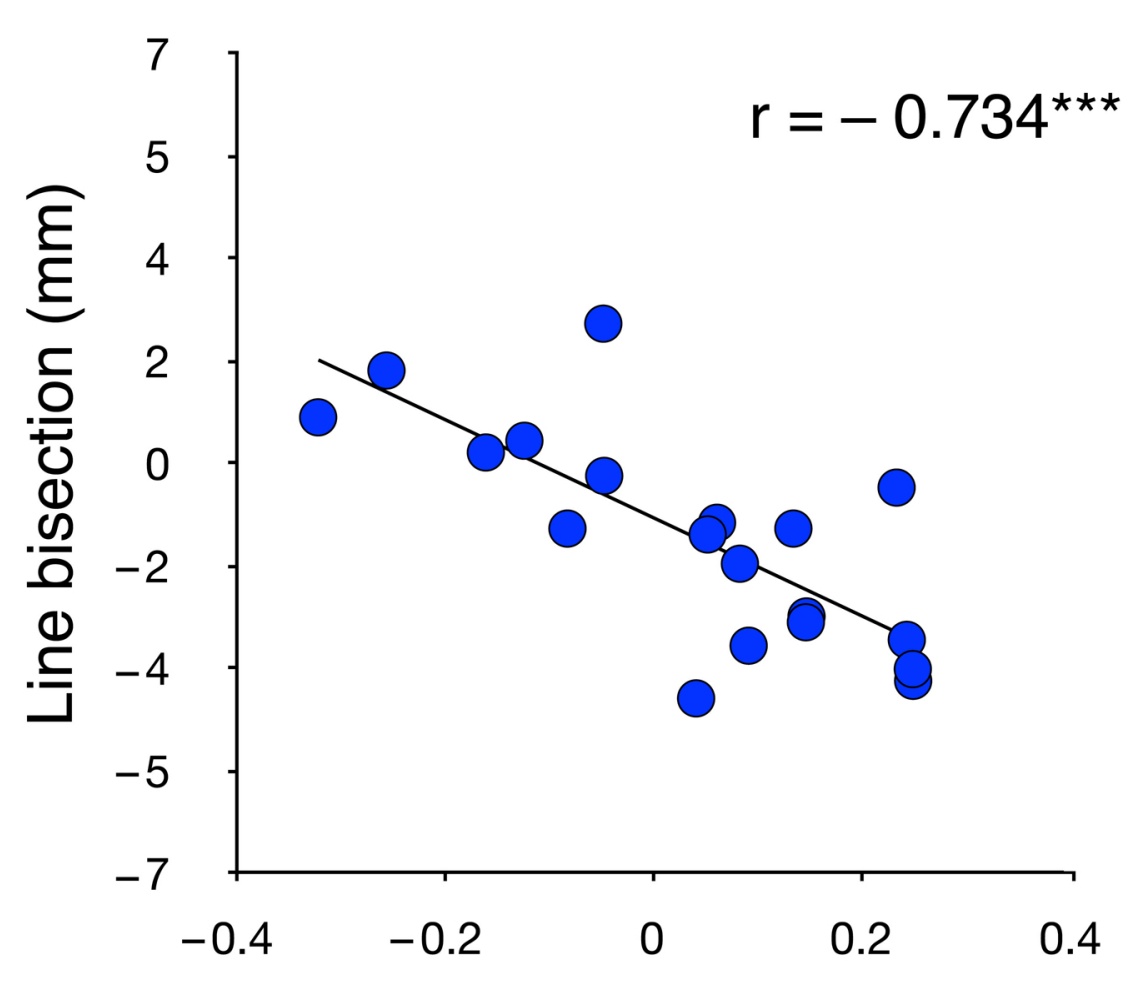

SLFII lateralization index

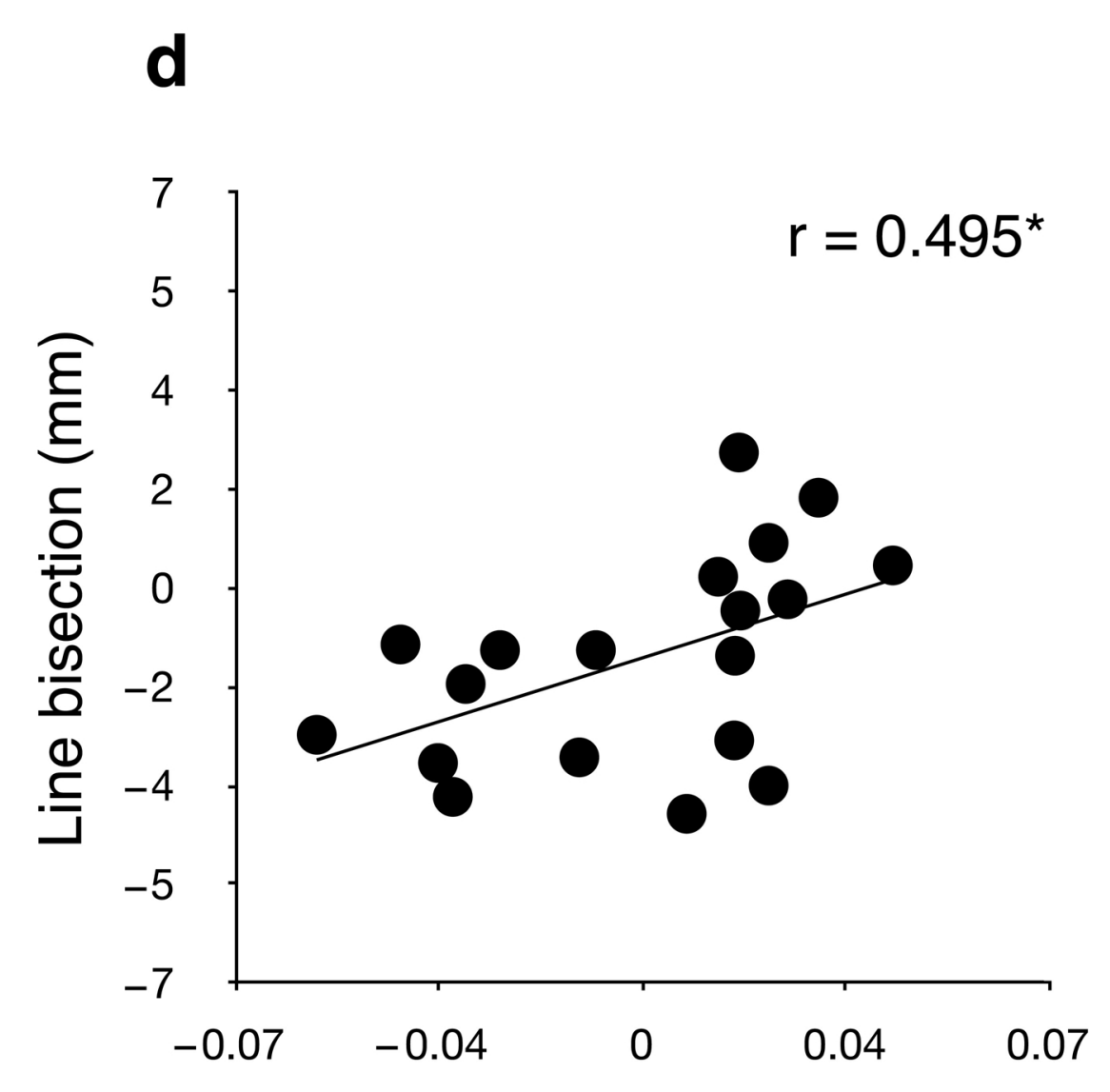

Lateralization index of detection time 